\title{
Structural Interrogation of Proteins Involved in the Biosynthesis of 10-Membered Enediyne Anticancer Natural Products.
}

\author{
Abstract \\ Abigael Kosgeia, Mitch Millerb, David Xuc ${ }^{\mathrm{c}}$, George N. Phillips ${ }^{\mathrm{d}}$ \\ a. Rice University, 6100 Main Street Houston, Texas 77005, ajk15@rice.edu \\ b. Rice University, 6100 Main Street Houston, Texas 77005, mitchm@rice.edu \\ c. Rice University, 6100 Main Street Houston, Texas 77005, wx7@rice.edu \\ d. Rice University, 6100 Main Street Houston, Texas 77005, georgep@rice.edu
}

Natural products are unparalleled resources for drug discovery. Most natural products owe their existence to the enzymatic machinery that runs inside microbes and plants. Through understanding the natural products biosynthetic pathway, we can exploit the catalytic ability of enzymes and use them as biocatalysts for structural diversification, activity optimization, and generation of previously unobserved natural products with novel antitumor functions.

Enediynes natural product has shown potential as anticancer antibiotics. They bind to the minor groove of DNA in such a way that they can abstract two hydrogen atoms from the sugars of the opposite strands causing double-stranded DNA cleavage. To ascertain the biochemical functionalities of proteins encoded by genes involved in the biosynthetic pathway of 10-membered ring enediynes, we utilize Xray crystallography to determine the atomic structures of these proteins, which shed a light to their functions.

My project is geared towards determining the atomic structures of CalU22, CalU17, DynF, and Orf14. CalU22 and CalU17 are proteins encoded by genes involved in the biosynthesis of 10-membered enediyne calicheamicin (CAL) while genes involved in the biosynthetic pathway of 10-membered enediyne dynemicin (DYN) encode proteins DynF and Orf14. The four target genes have been cloned and expressed but only DynF and CalU17 proteins were soluble. DynF and CalU17 proteins were purified using immobilized metal affinity chromatography with Ni-NTA resin and crystallized using sitting drop method.

From the X-ray diffraction data, CalU17 crystallized in the tetragonal space group $\mathbf{P 4}{ }_{3} \mathbf{2}_{1} 2$ while DynF crystallized in the orthorhombic space group C222 ${ }_{1}$. The phase problem was solved using SAD and molecular replacement for DynF and CalU17 respectively. The structures of CalU17 and DynF were solved. From the structures, the roles of CalU17 and DynF in the biosynthetic pathway of CAL and DYN will be elucidated. The structures of Orf14 and CalU22, along with their biochemical functions, are yet to be determined. The results will inform how 10-membered ring enediyne natural products are synthesized and will expand our abilities to modify and generate a library of novel small molecules that will be used for drug screening. 